\title{
SimNav: \\ Simulink Navigation of Model Clone Classes
}

\author{
Eric J. Rapos, Andrew Stevenson, Manar H. Alalfi, James R. Cordy \\ School of Computing, Queen's University \\ Kingston, Ontario, Canada \\ Email: $\{$ eric, andrews, alalfi, cordy $\} @$ cs.queensu.ca
}

\begin{abstract}
SimNav is a GUI designed for displaying and navigating clone classes of Simulink models detected by the model clone detector Simone. As an embedded Simulink interface tool, SimNav allows model developers to explore detected clones directly in their own model development environment rather than a separate research tool interface. SimNav allows users to open selected models for side-by-side comparison, in order to visually explore clone classes and view the differences in the clone instances, as well as to explore the context in which the clones exist. This tool paper describes the motivation, implementation, and use cases for SimNav.
\end{abstract}

SimNav can be downloaded here:

http://cs.queensu.ca/home/eric/SimNav.html

A video demonstrating SimNav can be found here: https://youtu.be/6oPR3U31I8M

\section{INTRODUCTION}

Clone detection in Simulink models can serve a number of purposes, such as finding model patterns and anti-patterns, examining evolution between versions, enforcing consistency and standards, and many other applications in model maintenance and quality assurance. Simone [2] is a text-based near-miss (Type 3) clone detector for Simulink model clones based on the NiCad [9] code clone detector. The native NiCad output of Simone, consisting of text-based HTML and XML reports, can be difficult for Simulink model developers to understand and relate to the visual Simulink graphical models they normally work with. SimNav provides a bridge between these two representations that is intended to bring the clone detection results of Simone directly into the developers' own Simulink modelling environment where they can explore, investigate and act upon clone detection results in the context of their own familiar workspace.

Emergent patterns in Simulink models found through clone detection [4], whether they are exact clones or near-miss clones, provide developers with useful information about a collection of models. Similar model sections can be indicative of the reuse of model sections, either intentionally or unintentionally, which can provide developers with the opportunity to create new library blocks, which can replace the clone instances. Model clones can also uncover instances of documented design patterns or anti-patterns [11], assisting in overall quality improvement.

Simone is also useful for studying evolution of Simulink models and model clones [10]. By using Simone on multiple versions of the same model, it is possible to identify sections of models that remain unchanged from version to version, or change minimally (near-miss results), as well as observing how clone classes evolve over versions. This information is useful as it reveals areas of models that remain consistent over time, and the inverse set can be used to find sections of the model that are prone to changes in each version, which is also useful for developers as it indicates sections that may be prone to the introduction of bugs in subsequent versions.

Each of these uses reveals information that can be used by developers to improve the model maintenance process. The detection of patterns can lead to the creation of library blocks for commonly occurring exact clones, and the detection of near-miss clones can assist in Simulink variability modelling [3]. The detection of clones over model versions reveals information that will help in the maintenance over time by identifying sections of models that require additional attention and testing, as they evolve more frequently than others, as well as sections that evolve less frequently, or not at all. SimNav assists in bringing the results of clone analysis directly into the environment where these tasks are carried out and actions can be taken to address issues directly.

The next section of this paper examines related approaches to presenting clone detection results. The remainder of the paper presents the implementation of SimNav, detailing the technical aspects, as well as presenting several use cases, accompanied by screen shots of the tool.

\section{RELATED WORK}

CloneDetective [6] is a tool capable of performing clone detection on Simulink models and presenting the results to users in a graphical interface. CloneDetective reproduces the models and displays them in a custom stand-alone tool, rather than directly within Simulink, which requires that developers learn a new interface and precludes the possibility of working with the models directly.

ModelCD [8] presents another model clone detection tool for Simulink models. ModelCD uses an algorithm called aScan to find approximate clones, which are analogous to Simone's near-miss clones, and is also capable of finding exact clones using another algorithm called eScan. ModelCD allows for the viewing of clones within Simulink using a Matlab script that opens and colours the clones in Matlab, but the actual clone detection results are not available in Simulink and the viewed models are copies of the originals. The functionality of ModelCD is somewhat similar to opening individual models using SimNav, but without the ability to explore clone classes, similarity and instances directly in the Simulink workspace.

The Naive Clone Detector [7] is an exact clone detector for Simulink models. Similarly to the other approaches discussed, 
the results are not presented directly in Simulink, but rather a Matlab Connector is provided. The Naive Clone Detector is capable of presenting the clone detection results, however the Matlab Connector must be used to separately open the models in Simulink.

Model Quality Assessor [5] is another tool used to view clone detection results (referred to by the authors as clone inspection). Model Quality Assessor is an external tool capable of visually displaying the clones that were detected, but lacks the direct integration with the Simulink environment provided by SimNav.

\section{IMPLEMENTATION}

One of the main goals of our implementation was the integration with the Simulink environment; our tool needed to be familiar to Simulink model developers without training. As such, all development was done directly in Matlab, making use of the various libraries and functionality provided.

The remainder of this section details specific design choices in the areas of user interface design, filtering and focussing methods, and outlines our process for industrial feedback.

\section{A. User Interface Design}

The SimNav user interface (UI) itself is simply designed to avoid cluttering and to ensure that users can easily identify how to perform desired tasks. The main portion of the UI is a table which displays the clone classes that were detected by Simone. The table has four columns: (i) Clone Class, (ii) Similarity, (iii) Subsystem, and (iv) Model File. The Clone Class number is a unique ID assigned to each clone class sequentially, and used to reference a clone class. The Similarity value indicates the similarity for a particular clone class as a percentage. The Subsystem identifies the full path the subsystem where the clone instance occurs. And finally, the Model File indicates which model file the subsystem is located in.

Beyond the table with its four columns, there are six controls along the top of the GUI: (i) Load Report, (ii) Open Selected, (iii) Close All, (iv) Deselect All, (v) Clone Type, and (vi) Similarity. Load Report will open a dialog box, which is used to select a Simone report to load into SimNav for further inspection. Open Selected will open all selected clone instances (instances are selected using a standard Ctrl+Click method, or by selecting an entire clone class by clicking on its ID) and tile them on the screen. Close All will close all opened models, and Deselect All will remove highlighting of any selected models. Alternatively, clicking on another clone class ID will close all open models, deselect all selected models, and select the new clone class. The Clone Type dropdown is used to filter the displayed results by the clone class type, which will be explained further in Section III-B. The Similarity control is used to filter the clone classes by their similarity; this is done by selecting a lower and upper bound on the similarity using the arrows on the selector boxes, and the filtering takes place after a 1 second delay, allowing the user to adjust both values without performing the sort multiple time. This filtering process will also be explained in Section III-B. Figure 1 shows all of these columns and controls in the SimNav toolbar.

Matlab's Graphical User Interface Design Environment (GUIDE) [1] provided us with the perfect platform for creating a user interface that would work within the Matlab/Simulink setting, while still allowing us the flexibility to implement our own functionality and features. GUIDE allowed us to create our UI using a simple drag and drop interface where we were able to add buttons and other controls, change their attributes, and assign function calls to any events where a response from the tool was necessary. The final UI for SimNav is simple, easy to navigate, and provides the desired functionality.

\section{B. Filtering}

SimNav allows for two types of filtering of the presented clone classes: (i) filtering based on the clone class type, and (ii) filtering based on the similarity of the clone class. This section will describe the implementation of these two types of filtering.

The first type of filtering is based on the concept of a clone type. As part of the refinement process, it became evident that the clone analysis would be useful to find clones within a single model, as well as across model files. As part of this, we developed the concepts of internal clone classes and external clone classes.

Internal clone classes are clone classes that contain only model instances from within the same model. Any clone class that has two or more instances from the same model file would be displayed during and Internal Only filter. An internal clone class may contain model instances from more than one model file, but each model file must have at least two instances, and any singleton model files are removed. As part of this filter, the model instances are sorted by model file name, in order to see how many instances occur in each file. For example, a clone class that originally contains two instances from Model A, one instance from Model B, two instances from Model C, and one instance from Model D, when filtered for internal clones, would only display the two instances from Model A and the two instances from Model $\mathrm{C}$, sorted such that the pairs are shown together.

External clone classes are clone classes that contain only model instances from different models. Any clone class that has at most one instance from a model file would be displayed during the External Only filter.

The default sort for SimNav is the Internal \& External sort, which shows both types, and all instances in each clone class.

The filtering based on clone class is done at the time of loading the report. When the report is loaded into SimNav, three tables are created - one for each of the types of sorts. The tables are constructed by iterating through the input file, and constructing the 4-column table that is displayed within the UI of SimNav. Each clone class is selected and all of the information is added to the table representing the default sort of Internal \& External, then the clone class is explored to see which of the other two types of filtering it matches, and is added to one of those tables as well. Once the three tables are stored in memory, the default filtering is displayed on the UI, and the other two are readily available for quick replacement, meaning that no matter how many times the view is changed, the filtering is only conducted once when the report is loaded.

The second type of filtering that SimNav is capable of is filtering based on the similarity of the clone class. The default filtering is from whatever the lower threshold of the particular set of models is, which is identified at load time, to $100 \%$. 


\begin{tabular}{|c|c|c|c|c|c|c|c|c|c|}
\hline \multicolumn{9}{|c|}{ SimNav v2.0 } & \multirow[t]{2}{*}{\begin{tabular}{|l|l|l|}
$口$ & 回 & $x$ \\
\end{tabular}} \\
\hline Load Report & Open Selected & Close All & Deselect All & Clone Type: & Internal \& External & $\checkmark$ & Similarity: & $70 \div-100 \div$ & \\
\hline Clone Class & Similarity & \multicolumn{4}{|c|}{ Subsystem } & \multicolumn{4}{|c|}{ Model File } \\
\hline
\end{tabular}

Fig. 1. The SimNav Toolbar

However it may be useful to filter out uninteresting results, such as those that are too similar or too dissimilar. Without wanting to impose too many restrictions on the filtering, we wanted it to be as free as possible, meaning that the user is able to choose a similarity range down to a granularity of a single percent.

Unlike the clone class type filtering, the similarity filtering is not done at the load time, and is done on the fly as the user selects the filtering they would like to be displayed. Once a user has selected a similarity range using the selector boxes, the upper and lower bound are passed to the filtering function to perform the filter, along with the current clone class filtering type. The filtering function will create a fresh copy of the full table for the currently selected clone class type (created at load time) and will iterate through each clone class, removing those that are outside of the set bounds. Once each clone class is either included or excluded, the resulting set of clone classes is displayed in the UI.

\section{Industry Feedback}

Throughout the development process, we collaborated with our industry partners, presenting iterations of the tool, and requesting feedback on both functionality and UI design. This section will detail some of the feedback and how it was incorporated into our design.

The first major piece of feedback that came from our industry partners was a request to be able to see only the clone classes that contain clones within a single model file. This was a use-case that we had not previously considered, which led to the introduction of the filtering based on clone class types.

Similarly, the other type of filtering, based on similarity, was also a result of feedback from our industry partners. Initially, we would only present all of the results, but it was communicated to us that it would be useful to exclude some of the results, some of the time, meaning that it would be good to allow the user to choose for themselves which ranges of similarity they wanted to display.

Beyond the two filters, our industry partners also provided us with minor feedback relating to UI design; a few requests that would make the UI more intuitive and responsive to the way work-flow would normally occur.

The collaboration with industry has provided us with additional insight into the use cases for SimNav which allowed us to further develop the tool into something desired for use in industry, and the positive feedback about the Simulink integration validated our initial goals.

\section{USE CASES}

The following use cases show basic functionality of SimNav, using a combined set of open-source automotive models, which we first ran Simone on to obtain clone detection results.

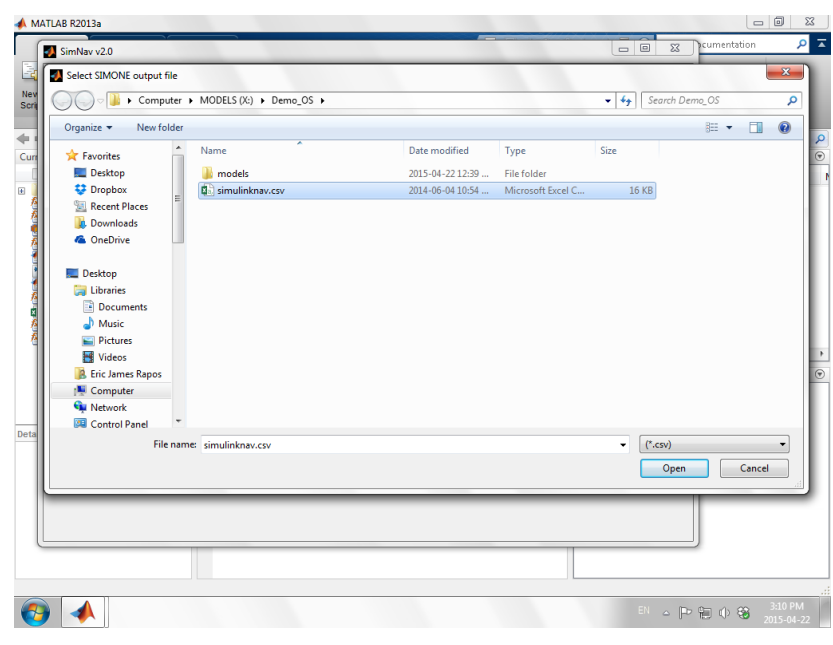

Fig. 2. Choosing the Simone Report to load

The models come from a number of open-source projects, and can be found on the SimNav page along with the download of SimNav.

\section{A. Viewing Clone Class Reports}

The first use case for SimNav is the viewing of the clone class reports generated by Simone. As part of the clone detection process, Simone produces a CSV file, which contains the clone classes, and the instance models. SimNav provides the functionality of choosing any of these reports using a simple file selection box, as seen in Figure 2.

After choosing a Simone report, SimNav loads up the clone classes and displays them in a table, providing the user with the following attributes: clone class ID, similarity, subsystem name, and model file. An example of a loaded report can be seen in Figure 3.

With this view, the user can examine how many clone classes were detected, the sizes of the clone classes, how similar the clone classes are, and which models and subsystems occur within the clone class.

\section{B. Viewing Clone Class Instances}

Once the user has identified the model clones of interest, they may select manually any number of models, or an entire clone class at a time by clicking on the clone class number. The selection of an entire clone class can be seen in Figure 4 for clone class 1 .

After clicking on Open Selected, the models are opened and the clone instances are tiled on the screen. Figure 5 shows the opening of clone class 1, tiling the two instances side-byside. Clone class 1 has a similarity of $83 \%$, meaning it is not exact, so a visual inspection of the models can help identify how the models differ. In Figure 5 we see that there is a minor 


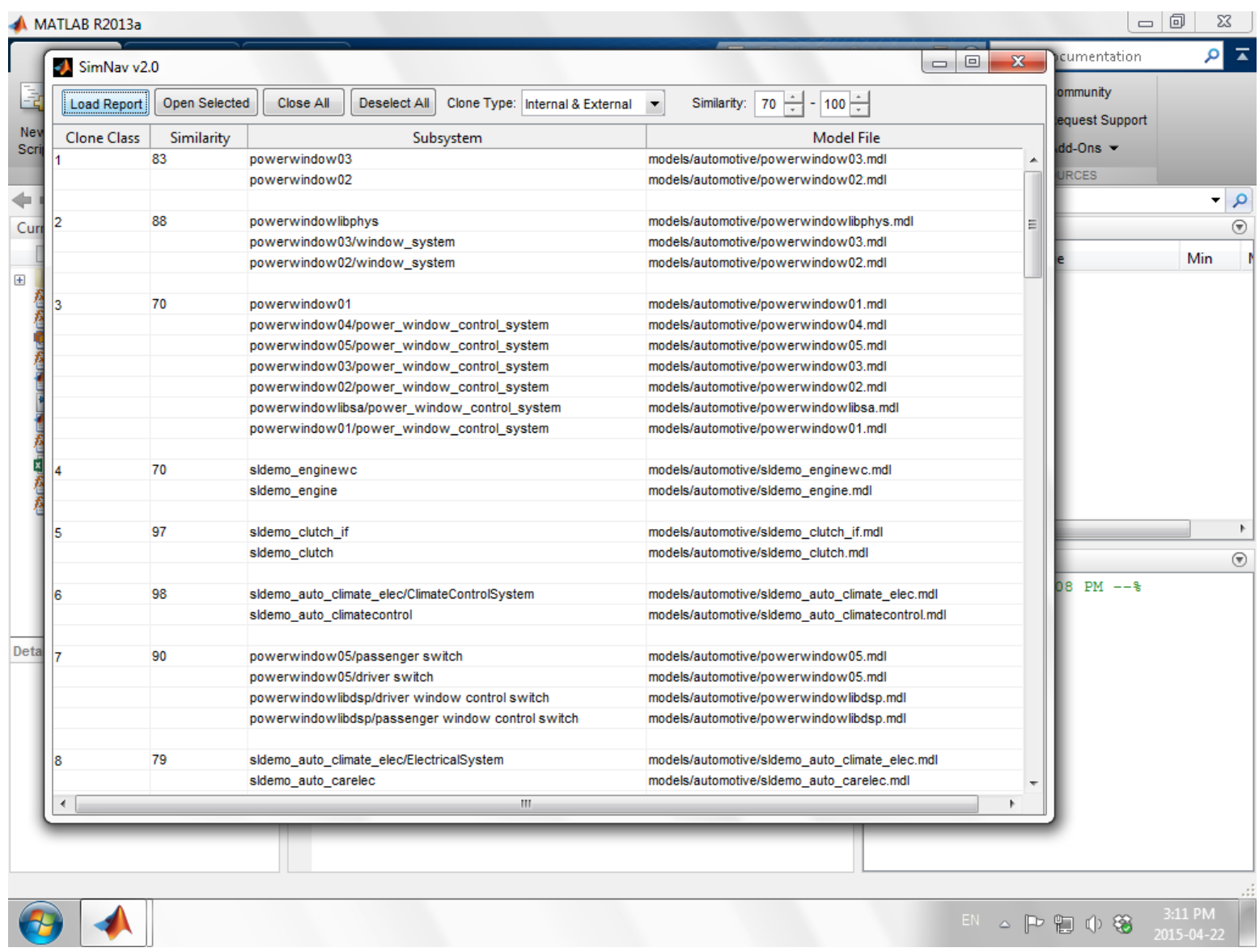

Fig. 3. Main view of SimNav

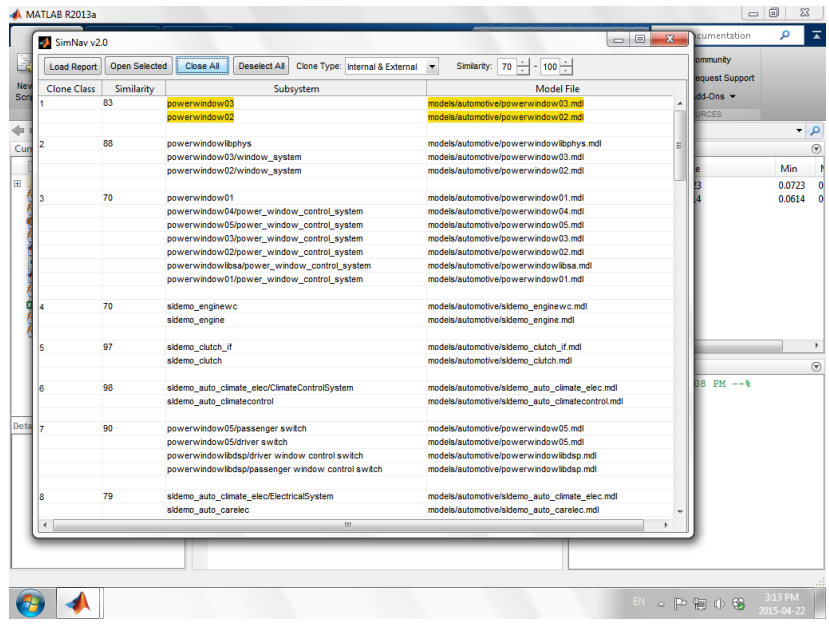

Fig. 4. Selecting all of clone class 1

difference at the top level view: the output block on the lower right hand side is different in each model (one is a subsystem and the other is a terminator block).

SimNav is capable of displaying more than two models at a time, which can be seen in Figure 6 which displays the opening of clone class 2 , which has three models, and a similarity of $88 \%$. This particular clone class reveals an interesting result, as it found a library block, and two instances of the library block over versions of the model.

Once done examining the models, SimNav is capable of closing all of the instances in two different ways: clicking the Close All button, or by selecting a new clone class. The first option will close all of the open models, but they will still be selected in the SimNav GUI, which can then be deselected using the Deselect All button. The second option will automatically close all open models, deselect those models, and select the new clone class.

\section{Filtering Results}

In addition to simply viewing the clone class results and opening models, SimNav provides the user with additional functionality related to filtering the clone class results.

As explained in Section III-B, SimNav implements two types of filtering. Using the same model set from the previous use case, and using the Internal Only filter, SimNav would provide the view in Figure 7 where a number of Clone Classes are filtered out $(1,2,4,5,6,8 \ldots)$.

The other type of filtering available in SimNav is based on the Similarity of the clone class, and wanting to remove any clone classes that may not be relevant to the current analysis. Figure 8 shows filtering the models to only display clone classes with similarity between $75 \%$ and $89 \%$, which leaves only five clone classes $(1,2,8,10$, and 11). 


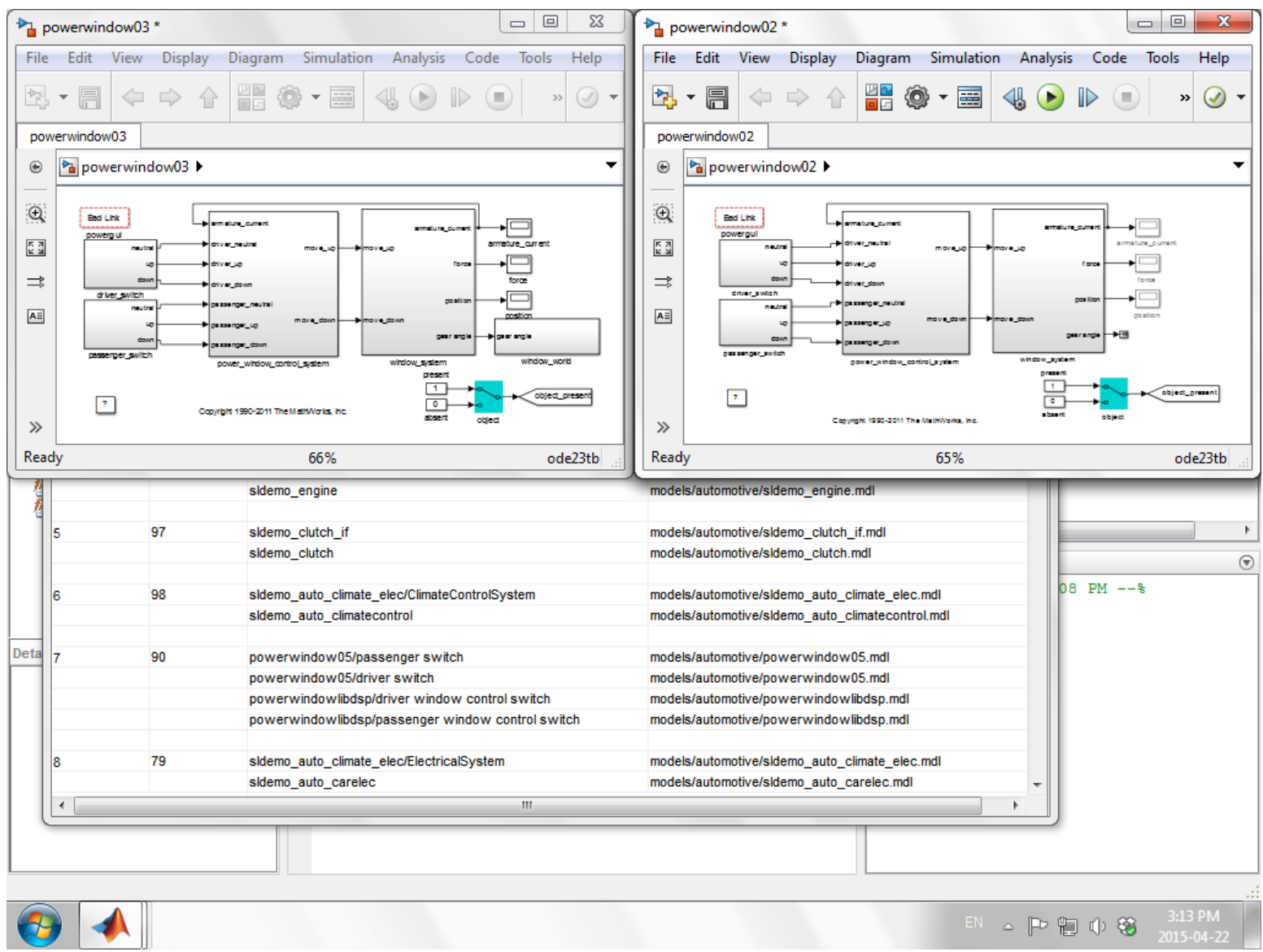

Fig. 5. Opening clone class 1 , automatically tiled ( 2 models)

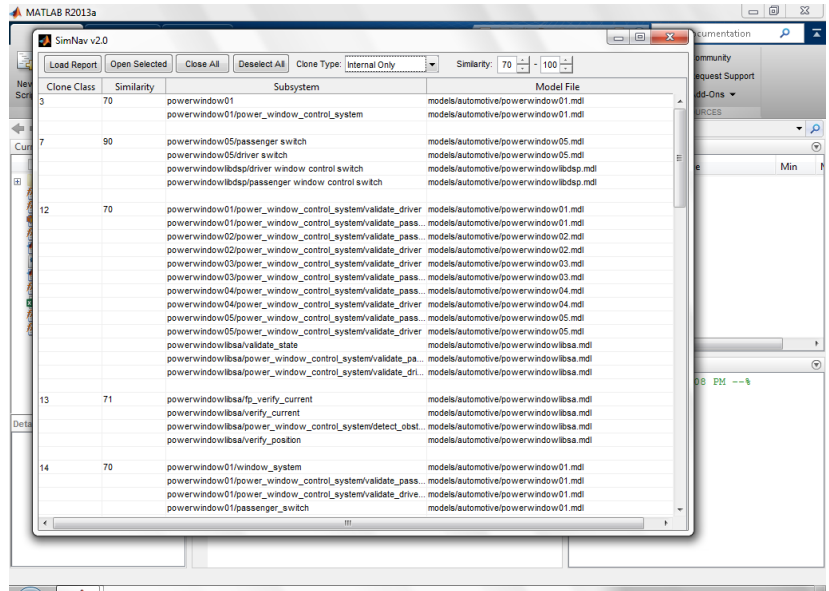

91

Fig. 7. Filtering results to show only clone classes with internal clones

\section{FUTURE WORK}

There are still several features that we would like to include in SimNav which would increase its usefulness.

The first of these would be the ability to visually identify differences and similarities within a clone class. Currently, users need to manually identify these by looking at instances, but we plan to implement a method of colouring differences

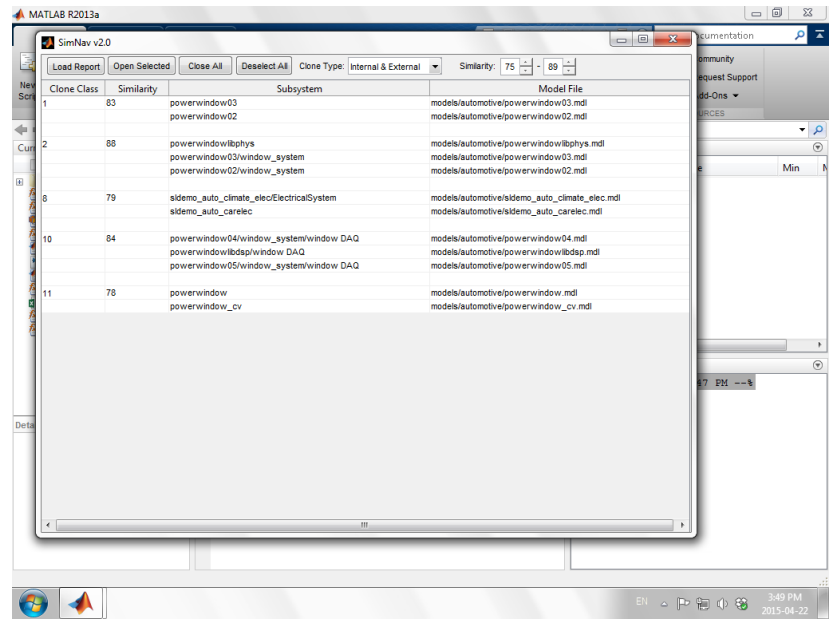

Fig. 8. Filtering results to show only clone classes with similarity between $75 \%$ and $89 \%$

and/or similarities, to easily show how models differ. We have begun early work on this feature, which is relatively simple for pairs of models, but increases in complexity as the clone class increases in size.

Another use case for SimNav is the ability to create variability models of near-miss clone classes [3]. The ability for SimNav to take a clone class, which is mostly similar, with a 


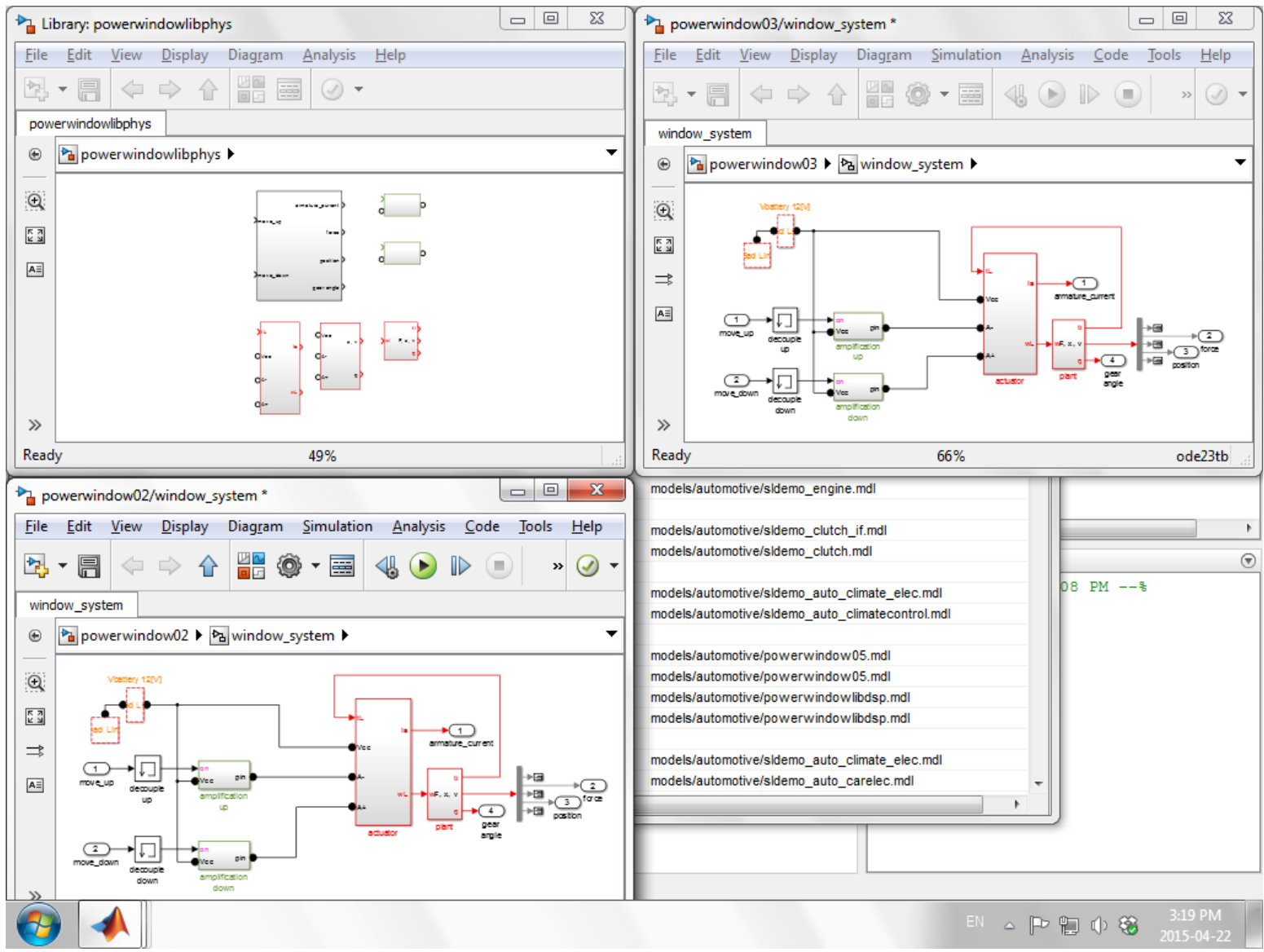

Fig. 6. Opening clone class 2, automatically tiled (3 models)

few minor differences, and merge them into a single variability model, would allow for the automation of previous work on variability and model product lines, and further improve the maintenance of Simulink models.

\section{CONCLUSION}

SimNav implements the presentation of results from the Simone model clone detector directly in the Simulink environment, a property absent from many other clone detection tools for Simulink models. The ability to view results and open models within Simulink makes SimNav an effective tool for navigation of Simulink clone classes. Further the filtering features of SimNav add to its usefulness to developers, allowing them to display only clones of interest to them in a particular use case, whether that is clones of a certain type or within a certain similarity range. The industry collaboration involved in the development of SimNav has been a huge asset.

\section{ACKNOWLEDGMENTS}

The authors would like to thank the NECSIS Automotive Research Network, General Motors, and NSERC for their support. Additionally, we would like to thank Kenny-Luc Vuong, Melanie Wightman, and Ron Elbaz for their contributions.

\section{REFERENCES}

[1] Mathworks MATLAB GUI Design. http://www.mathworks.com/ discovery/matlab-gui.html. Accessed: 2015-05-21.

[2] M.H. Alalfi, J.R. Cordy, T.R. Dean, M. Stephan, and A. Stevenson. Models are code too: Near-miss clone detection for Simulink models. In ICSM 2012, pages 295-304, Sept 2012.

[3] M.H. Alalfi, E.J. Rapos, A. Stevenson, M. Stephan, T.R. Dean, and J.R. Cordy. Semi-automatic identification and representation of subsystem variability in simulink models. In ICSME 2014, pages 486-490, 2014.

[4] J.R. Cordy. Submodel pattern extraction for Simulink models. In SPLC 2013, pages 7-10, 2013

[5] F. Deissenboeck, B. Hummel, E. Juergens, M. Pfaehler, and B. Schaetz. Model clone detection in practice. In IWSC 2010, pages 57-64, 2010.

[6] E. Juergens, F. Deissenboeck, and B. Hummel. CloneDetective-a workbench for clone detection research. In ICSE 2009, pages 603606, 2009

[7] H. Petersen. Clone detection in matlab simulink models. Master's thesis, Technical University of Denmark, 2012.

[8] N.H. Pham, H.A. Nguyen, T.T Nguyen, J.M. Al-Kofahi, and T.N Nguyen. Complete and accurate clone detection in graph-based models In ICSE 2009, pages 276-286, 2009.

[9] C.K. Roy and J.R. Cordy. NICAD: Accurate detection of near-miss intentional clones using flexible pretty-printing and code normalization In ICPC 2008, pages 172-181, 2008.

[10] M. Stephan, M.H. Alalfi, J.R. Cordy, and A. Stevenson. Evolution of model clones in Simulink. In ME@ MODELS 2013, pages 40-49, 2013.

[11] M. Stephan and J.R. Cordy. Identifying instances of model design patterns and antipatterns using model clone detection. In MISE 2015 , pages 48-53, 2015. 